\title{
Randomised controlled trial of the use of a modified postal reminder card on the uptake of measles vaccination
}

P Hawe, N McKenzie, R Scurry

\begin{abstract}
Objective-To determine whether rewording postal reminder cards according to the "health belief model", a theory about preventive health behaviour, would help to improve measles vaccination rates.

Design-A randomised controlled trial, with blind assessment of outcome status. Parents of children due for their first measles vaccination were randomised to one of two groups, one receiving the health belief model reminder card, the other receiving the usual, neutrally worded card. The proportion of children subsequently vaccinated in each group over a five week period was ascertained from clinical (provider based) records.

Setting-A local government operated public vaccination clinic.

Participants-Parents of 259 children due for measles vaccination.

Main results-The proportion of children vaccinated in the health belief model card group was $79 \%$ compared with $67 \%$ of those sent the usual card $(95 \% \mathrm{CI}, 2 \%$ to $23 \%$ ), a modest but important improvement.

Conclusion-This study illustrates how the effectiveness of a minimal and widely practised intervention to promote vaccination compliance can be improved with negligible additional effort.

(Arch Dis Child 1998;79:136-140)
\end{abstract}

Keywords: measles vaccination; postal reminders; randomised controlled trial; health promotion

Studies of postal reminder systems for vaccination have appeared in the literature since the early $1960 \mathrm{~s},{ }^{1}$ and the impact of computer assisted registries has been evaluated in the $\mathrm{UK},{ }^{2}$ Canada, ${ }^{3}$ and New Zealand. ${ }^{4}$ Recently, a national computer based registry and postal reminder system has been established in Australia, as part of a renewed national strategy to improve childhood vaccination rates. ${ }^{5}$ Increasingly, however, the call for evidence based practice has led to a re-examination of many widespread practices in both health care and public health. In childhood vaccination, a systematic review of parent level interventions (such as reminder systems) by Tannenbaum and his colleagues in 1994 concluded that there was insufficient evidence to recommend that they be routine practice for measles/ mumps/rubella vaccination or diphtheria/ pertussis/tetanus vaccination. ${ }^{6}$ This was be- cause of the relatively weak methods used in most studies up until that time and insufficient accumulation of evidence from the few studies that were rated as moderate or strong. However, a most striking feature of previous evaluations of childhood vaccination reminders is the extent to which they have ignored theory and research in health behaviour. Researchers have not given much thought to the way that messages should be framed. Indeed, very few studies have reported the content of the messages, with a notable exception being the study by Stehr-Green et al pertaining to telephone reminders. ${ }^{7}$ We would argue that by failing to appraise critically the content of the reminders along with the quality of the study design, systematic reviews in vaccination have given us only a partial view of the potential of particular interventions. The design of preventive interventions is not "common sense". Indeed, there is a possibility that so-called motivational mailings about vaccination, designed in ignorance of theories about health behaviour, ${ }^{8}$ might in fact be counter productive. ${ }^{9}$ One popular health behaviour theory is the "health belief model". It states that people will be more likely to undertake a health behaviour if they feel that: (1) the disease in question is severe, (2) they are susceptible to it, and (3) the action that is recommended will have benefits that will outweigh any associated costs. ${ }^{10}$ The health belief model was developed in the early 1950s by social psychologists in the US Public Health Service attempting to explain the widespread failure of people to participate in preventive health programmes, particularly $x$ ray screening programmes in tuberculosis control. ${ }^{11}$ Extensively researched since that time, its predictive validity in relation to a wide range of health behaviours has been the subject of review $^{12}$ and meta analysis. ${ }^{13}$ High quality intervention studies with the health belief model have been relatively few. ${ }^{13}$ Although there is some limited evidence that vaccination reminders based on the health belief model could increase adult vaccination rates, ${ }^{14}$ no researchers appear to have tested the usefulness of the model in relation to any of the routine childhood vaccinations. We set out to determine whether postal reminders reworded according to the health belief model could improve vaccination rates for measles.

\section{Methods}

The study was conducted with a municipal council within the provincial city of Ballarat (regional population, 70 000). The council had 
Ballarat City Council

Dear Mrs QuINN,

MEASLES is still a problem in Ballarat, particularly for children under the age of 2 years. Some children suffer severe complications.

The children who are most likely to catch measles are those who have not been immunised. Immunisation is very effective. There is almost no chance of side effects. Clinics are held at the Lower Civic Hall in Mair Street from 2 - 3.40 pm EVERY SECOND WEDNESDAY. Immunisations are given by a doctor. Of course, imraunisation is free!

The next clinic is on Wednesday 14 th December. If GEORGIA is not yet immunised against measles, you should bring her along.

Regards,

Bob Scurry

HEALTH DEPARTMENT

Enquiries: ph 313277

Figure 1 Health belief model card.

\begin{tabular}{|c|}
\hline 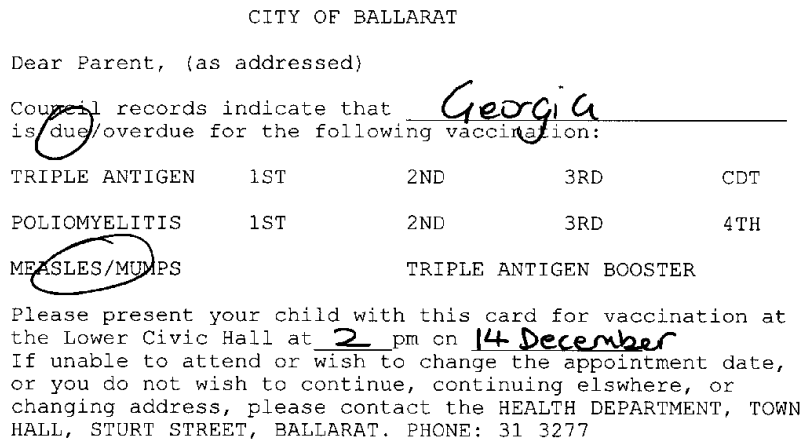 \\
\hline
\end{tabular}

Figure 2 Usual vaccination reminder card sent by the council.

an existing computerised reminder system. The content and main features of the health belief model card were derived from the literature ${ }^{12-14}$ We decided that the use of a no intervention control group, rather than a usual intervention control group, might yield a greater margin of difference compared with the experimental health belief model intervention, but that this would be an undesirable, artificial test scenario, given that postal reminder cards systems were widespread. Thus, the question being asked in our trial was not: "Shall we do ' $\mathrm{X}$ ' or shall we do nothing?" but rather: "What can we add to existing practice to improve health outcomes?" A series of four focus groups was conducted to pretest the health belief model card. These were held with parents from the target group considered to be hardest to reach with written messages - that is, people with low socioeconomic status, and minimal education. Parents were recruited for the focus groups by social and welfare workers in the surrounding municipalities - that is, areas outside the one within which the trial was to be conducted. As a result of these groups, the health belief model card was altered in a number of ways. Words such as "susceptible" were dropped because of parents' limited understanding. The title of the person who sends the card (the Chief Health Surveyor) was also dropped because it was viewed as intimidating. The card was signed from the "Health Department" instead. The final card was addressed specifically to the parent ("Dear Mrs Quinn" instead of "Dear Parent") and the child was referred to by name. The final format and wording of the health belief model card is illustrated in fig 1 . The usual neutrally worded card sent by the council clinic appears in fig 2 .

The study population comprised the parents of children born consecutively between 24 August 1987 and 28 February 1988, who were due for measles vaccination-that is, the children who reached 15 months of age during the study period (the recommended age for vaccination in Australia has since been reduced to 12 months of age). Children were identified from the municipal council lists, which are based on birth notifications supplied by local hospitals. The lists are thought to cover at least $98 \%$ of children in this age group in the municipality. ${ }^{15}$ The birth dates listed above encompassed 300 children, although it was agreed in advance that a single interim analysis would be conducted when approximately 262 children had been recruited into the study, and the trial stopped if a difference was significant at the $5 \%$ level. This sample size calculation was based on an expectation of the difference between the "health belief model" card and the usual card being in the order of $15 \%$, drawing on the results of a pilot study of 60 children and estimating the vaccination rate in the control group to be $70 \%$ (significance level of $5 \%$ and power of $80 \%) .{ }^{16}$ Parents of the 300 children due to receive measles vaccination reminders were allocated randomly into two groups, one to receive the usual card and one to receive the health belief model card. After randomisation and before cards were sent, the council was notified of the death of one child and two further children were vaccinated early by private medical practitioners. These three children were excluded from the analysis. After cards were sent to the remaining children, 10 cards were returned because the addresses were incorrect, or the family had moved. Nine cards were not sent because of administrative error. Cards were sent in batches according to when a child became due for vaccination. A vaccination clinic was held one week after cards were sent. The next clinic was two weeks later. Parents who did not have their children vaccinated at either of these first two clinics were sent a second reminder card, which was the same type as the first card they had been sent. Another clinic was held a week after the second card had been sent. After this time the final proportion of children who had been vaccinated in both groups was determined. A child was coded as "vaccination having been received" if the parent presented to any of the three clinics held after the first reminder card had been sent. We were also advised, by usual reciprocal arrangement with neighbouring councils, if any of the parents in our study group had their child vaccinated by a neighbouring council. We were also aware if a child had been vaccinated during this period by a private medical practitioner either because: (1) a parent volunteered this information upon receipt of our second reminder (and the medical practitioner could be named and the report verified); or (2) because the medical practitioner had advised us directly, as part of the usual arrangement 
Table 1 Sociodemograhic characteristics of study groups

\begin{tabular}{|c|c|c|c|c|}
\hline & $\begin{array}{l}\text { HBM card } \\
(n=90)\end{array}$ & $\begin{array}{l}\text { Usual card } \\
(n=83)\end{array}$ & $\begin{array}{l}\text { Difference } \\
(95 \% C I)\end{array}$ & $p$ value \\
\hline $\begin{array}{l}\text { Mean number of children in } \\
\text { family }\end{array}$ & 2.1 & 2.3 & $-0.2(-0.12,0.55)$ & 0.21 \\
\hline $\begin{array}{l}\text { Mean number of children } \\
\leqslant 5 \text { years }\end{array}$ & 1.6 & 1.8 & $-0.2(-0.05,0.32)$ & 0.14 \\
\hline Mean mother's age & 29.8 & 30.0 & $-0.2(-1.28,1.8)$ & 0.74 \\
\hline $\begin{array}{l}\text { Mean number of years mother at } \\
\text { high school }\end{array}$ & 4.9 & 4.6 & $0.3(-0.6,0.03)$ & 0.072 \\
\hline Mother born in Australia & $96 \%$ & $92 \%$ & $4 \%(-3,11)$ & 0.22 \\
\hline Mean father's age & 33.1 & 32.2 & $0.9(-2.7,0.97)$ & 0.35 \\
\hline $\begin{array}{l}\text { Mean number of years father at } \\
\text { high school }\end{array}$ & 4.6 & 4.5 & $0.0(-0.45,0.33)$ & 0.77 \\
\hline Father born in Australia & $91 \%$ & $90 \%$ & $1 \%(-8,11)$ & 0.78 \\
\hline Single parent family & $12 \%$ & $5 \%$ & $7 \%(-1,15)$ & 0.08 \\
\hline Dependent on pension/benefit & $13 \%$ & $9 \%$ & $4 \%(-6,13)$ & 0.45 \\
\hline
\end{tabular}

HBM, health belief model.

regarding distribution of vaccines by the council to doctors and agreement by doctors to report who they vaccinate to the council. The administrative assistant recording the vaccinations was not aware of what type of card a parent had received. At the conclusion of the trial, all parents in the trial sample were sent a self administered questionnaire to determine their sociodemographic characteristics. This step was taken at the end of the study rather than at the beginning to avoid the possibility of sensitising parents about the vaccination clinic and the Health Department before the postal trial was conducted. It was explained that the purpose of the questionnaire was to find out parents' views about the quality of the vaccination clinics and mode of transport used to get to clinics. This generated useful information secondary to the study purpose. The sociodemographic questionnaire included a number of sensitive questions (household composition and dependency on social security payments) and, as such, the decision was taken that it should be completed and returned anonymously, in order to maximise response rates. The only identifying information on the questionnaire was a symbol that identified the

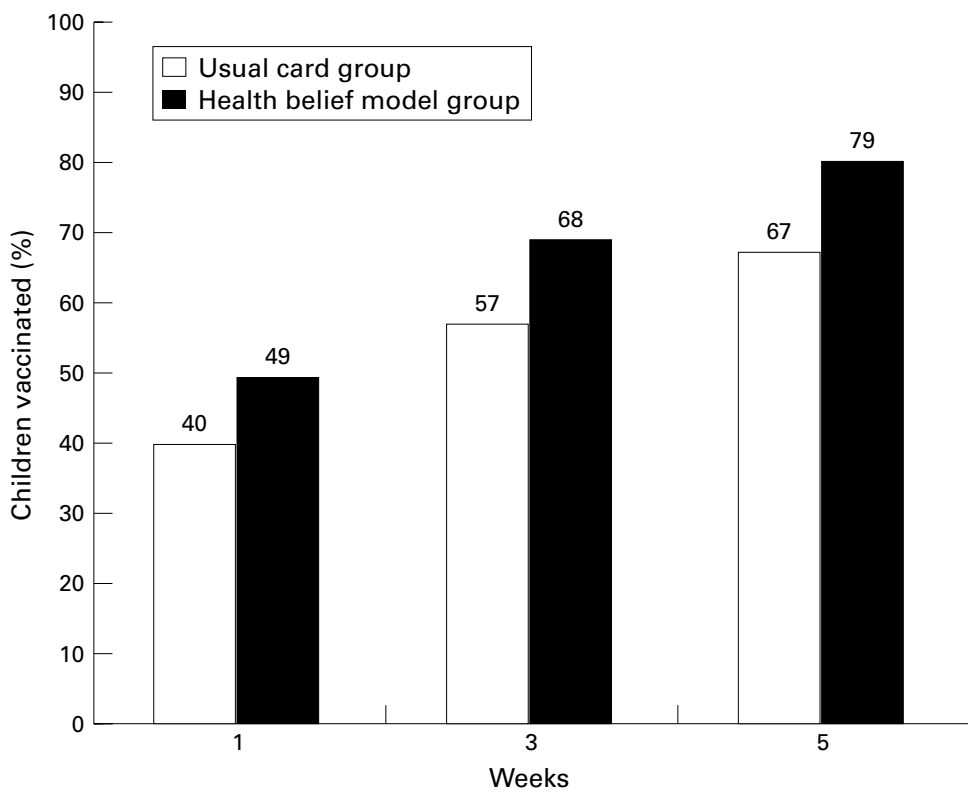

Figure 3 Percentage of children vaccinated in the five weeks following a postal reminder prompt. group membership of the respondent. This decision meant that personally identifying sociodemographic information could not be linked to vaccination rates in the subsequent analysis. Ethics approval for the study was received from the University of Melbourne. The analysis was conducted when 259 children had been recruited into the study, 124 into the health belief model card group and 135 into the usual card group. This was just short of the intended number because of failure to move the cut off point to compensate for the three excluded children (an error). The analysis was conducted using the principle of intention to treat-all eligible subjects were included in the analysis. The 10 children whose cards were returned because the parents had moved or the cards were not correctly addressed were considered "late ineligibles"17 and were included in the analysis. The nine children whose cards were not sent because of administrative error were ruled eligible and were included in the analysis. Two sample $t$ tests and $\chi^{2}$ analyses were conducted using the Minitab statistical package. The $95 \%$ confidence intervals to test the differences between the proportions were calculated using the formula supplied by Bland. ${ }^{18}$

\section{Results}

The sociodemographic characteristics of the two groups in the study are illustrated in table 1. One hundred and seventy three questionnaires $(67 \%)$ were returned after two attempts. Seventy one per cent of questionnaires were returned from the health belief model card group and $62 \%$ of questionnaires were returned from the usual card group. Apparent differences between the groups in table 1 (which are not significant) might possibly reflect a difference in those who returned the questionnaire rather than real differences between the groups, although the direction of bias, if any exists, is against the health belief model group. No additional data sources were available to determine whether the sociodemographic characteristics of those parents who returned the questionnaire differed from those who did not. The sample was almost entirely Australian born, a result that would be expected for a large country town in Australia, but would be unusual for large metropolitan areas. Other countries of birth represented in the sample include the UK, the Netherlands, Yugoslavia, Hong Kong, and Vietnam.

In the assessment of outcome, the 10 children who were lost to follow up (five in the health belief model group and five in the usual card group), for whom vaccination status was not known, appeared in the denominator and were counted as unvaccinated in the numerator (that is, the worse case scenario was assumed). The nine children whose cards were not sent were included in the denominator, and were included in the numerator according to their vaccination status. Three children in the health belief model group did not receive cards, one of whom was vaccinated, and six children in the usual card group did not receive cards, one of whom was vaccinated. The effectiveness of the 
health belief model card compared to the usual card is presented in fig 3. At the conclusion of the study, $79 \%$ (98 children) of the "health belief model" card group were vaccinated compared with $67 \%$ (90 children) of the usual card group. This is a difference of $12 \%$, which is significant $\left(95 \% \mathrm{CI}, 2 \%\right.$ to $23 \%$; $\chi^{2}=4.967$; $\mathrm{df}=1 ; \mathrm{p}=0.026)$. The significance of the difference between the groups was not sensitive to alternative handling of the 19 children who did not receive reminder cards. That is, the results remain significant with the 10 children with incorrect addresses removed $(\mathrm{n}=249$; $\left.\chi^{2}=5.784 ; \mathrm{df}=1 ; \mathrm{p}=0.016\right)$, or with all 19 removed from the analysis ( $\mathrm{n}=240$; $\left.\chi^{2}=4.824 ; \mathrm{df}=1 ; \mathrm{p}=0.028\right)$.

\section{Discussion}

In this study, a $12 \%$ improvement in vaccination rate was seen when a health belief model vaccination reminder card was used instead of the usual card sent by the council. However, it should be noted that the additional contribution of wording the card personally to the parent and signing it personally by a council officer might have enhanced the effect. That is, the effect size of the health belief model seen here is confounded by the additional effort made to personalise the card. Also, in the health belief model group, parents were given slightly more information about the location of the vaccination clinic, although the effect of this is considered to be negligible, given that all parents had visited the clinic up to 12 months before to receive the triple antigen vaccine. We took the view that the purpose of the trial was to maximise the effect of a postal reminder card, not to test the health belief model in a pure sense, and hence factors that added to the card's appeal were included. Although a significant $12 \%$ improvement was seen with the health belief model card, because the trial was stopped when the number of participants reached 259, the estimate of effect was less precise. A larger sample size would have narrowed the confidence intervals around the estimate of effect. The council was not keen to continue the trial once a clear effect had been shown for two reasons. The first was the desire to put the new card (if successful) into place, the second was to cut short the disruption caused by the additional workload of data collection. Nine cards were not sent in this trial because of administrative error. Incorrect or out of date addresses also thwarted the potential of the strategy being tested. Hence, the size of the effect observed here was diluted, and could have been higher. Registries will vary in the extent to which lists are up to date and in the degree of administrative attention they receive for upkeep of reminder systems. Hence, the effect of the card will vary in different settings. Stehr-Green and colleagues ${ }^{7}$ noted a similar problem in their randomised trial of a telephone computer generated reminder for childhood vaccinations. One fifth of their inner city sample could not be reached by telephone. The primary analysis by intention to treat showed a slight effect of only marginal significance, but an analysis among the sub- group who were reached by the telephone call showed a significant difference of $11 \%$ between the experimental and the (no intervention) control groups. A similar reinvestigation in our study of those who were known to have received cards (that is, excluding 19 children) adjusts the vaccination level in the experimental group from $79 \%$ to $84 \%$ and the corresponding levels in the control group from $67 \%$ to $72 \%$. However, it should be emphasised that such analyses effectively remove the "real life" conditions to which results of trials actually should be generalised and, as such, these types of analyses are generally discouraged. ${ }^{19}$

The differential response rate to the follow up questionnaire is difficult to interpret. We can only speculate that the parents receiving the health belief model card noticed and appreciated the card, were more likely to act upon it, and subsequently took more interest in returning a questionnaire about their opinion on council run services. The groups did not differ in the types of comments made about clinic services - that is, whether their remarks were positive or negative. The study population for this trial was predominantly Australian born. Although other investigators have shown that the health belief model is also a useful framework for analysing childhood vaccination behaviour in cross cultural contexts, ${ }^{20}$ we believe that the results here cannot be extrapolated to different ethnic groups. Appropriate formative research would be required to develop tailor written vaccination messages in other languages. In our study, $21 \%$ of the parents who received the health belief model card did not respond to the invitation to vaccinate their child in the following few weeks. Clearly, a range of factors affect vaccination compliance, only some of which can be addressed simply by increasing the sophistication of our written messages about vaccination importance. However, it is significant that the study has shown that with negligible additional effort, the effectiveness of a widely established strategy to promote vaccination compliance can be improved. Other investigators have also shown that the health belief model might be used to provide greater improvements in health outcomes in preventive medicine, ${ }^{13}$ although it should be noted that the apparent margin of benefit is likely to vary according to what the control group receives and according to situational conditions. For example, with regard to influenza vaccination reminders to parents of children with asthma, Szilagyi et al found a very large margin of difference conferred by the health belief model, in that $30 \%$ of children whose parents received a health belief model card were vaccinated compared with just $7 \%$ in a no intervention control group. ${ }^{21}$ Compared with many other countries, high routine childhood vaccination levels are already being achieved in the UK (although population pockets with lower vaccination rates might remain)..$^{22}$ In such circumstances, the degree of benefit that could be conferred by rewording vaccination postal reminders according to the health belief model is uncertain. However, complacency about vaccination 
levels should be avoided. Because universal vaccination is the goal, all possibilities for improvements on the margin, with minimal additional cost, are worthy of consideration.

This study was carried out with assistance from a National Health and Medical Research Council Public Health Research Fellowship granted to the first author and a Measles Immunisation Grant from the Victorian Department of Health.

1 Galloway T. Management of vaccination and immunisation procedures by electronic computer. Med Officer 1963;109:232-3.

2 Bussey AL, Harris AS. Computers and the effectiveness of the measles vaccination campaign in England and Wales. Commun Med 1979;1:29-35.

3 Loeser H, Zvagulis I, Hercz L, Pless B. The organisation and evaluation of a computer assisted centralised immunisation registry. Am F Public Health 1983:74:1298-300.

4 Reid JS, Graham-Smith HJ. Childhood immunisations: recall system is worthwhile. NZ Med f 1984;97:688-9.

5 National Health and Medical Research Council. National immunisation strategy Canberra: Australian Government Publishing Service, 1993.

6 Tannenbaum TN, Gyrokos T, Abrahamowicz M, et al. Immunisation delivery methods: practice recommendations. Can f Public Health 1994;1 (suppl):S37-40.

7 Stehr-Green PA, Dini EF, Lindegren ML, Patriarca PA. Evaluation of telephone computer-generated reminders to Evaluation of telephone computer-generated reminders to
improve immunisation coverage at inner city clinics. Public improve immunisation coverage

8 Byrne EB, Schaeffer W, Dini EF, Case GE. Infant immunisation surveillance: cost vs effect. $\mathcal{F} \mathrm{Am} \mathrm{Med} \mathrm{Assoc}$ 1979;212:770-3.

9 Job RFS. Effective and ineffective use of fear in health promotion campaigns. Am F Public Health 1988;78:163-7.

10 Becker M, ed. The health belief model and personal health behaviour. Health Educ Monogr 1974;2:324-508.
11 Rosenstock IM. The health belief model: explaining behaviour through expectancies. In: Glanz K, Lewis FM, Rimer $\mathrm{BK}$, eds. Health behaviour and health education. Theory, research and practice. San Francisco: Jossey Bass, 1990:3962.

12 Janz NK, Becker MH. The health belief model. A decade ater. Health Educ Q 1984;11:1-47.

13 Harrison JA, Mullen PD, Green LW. A meta-analysis of studies of the health belief model with adults. Health Educ Res 1992;7:107-16.

14 Larson EB, Bergman J, Heidrich F, Alvin BL, Schneeweiss R. Do postcards improve influenza vaccination compliance? Med Care 1982;20:639-48.

15 Thompson S. Programme management: immunisation in Victoria. Address to the 3rd National Immunisation Conference Public Health Association of Australia. Melbourne, April 1993.

16 Casagrande T, Pike MC, Smith PG. The power function of the exact test for comparing binomial distributions. Applied statistics 1978;27:176-80.

17 Pocock SJ. Clinical trials. A practical approach. New York: John Wiley and Sons, 1985.

18 Bland M. An introduction to medical statistics. Oxford: Oxford Medical Publications, 1991.

19 Newell DJ. Intention to treat analysis: implications for quantitative and qualitative research. Int 7 Epidemiol 1992; 21:837-41.

20 Eng E, Naimoli J, Naimoli G, Parker KA, Lowenthal N. The acceptability of childhood immunization to Togolese mothers: a sociobehavioural perspective. Health Educ $O$ 1991;18:97-110.

21 Szilagyi PG, Rodewald LE, Savageau J, Yoos L, Doane C. Improving influenza vaccination rates in children with asthma: a test of a computerised reminder system and an analysis of factors predicting vaccination compliance. Pedianalysis of factors pre
atrics $1992 ; 90: 871-5$.

22 Reading R, Colver A, Openshaw S, Jarvis S. Do interventions that improve immunisation uptake also reduce inequalities in uptake? BMf 1994;308:1142-4. 